Z Gerontol Geriat 2015 - 48:103-104 DOI 10.1007/s00391-015-0865-4

Online publiziert: 30. Januar 2015

๑) Springer-Verlag Berlin Heidelberg 2015

\author{
Werner Hofmann ${ }^{1}$ • U. Sommeregger ${ }^{2}$. S. Krupp ${ }^{3}$ \\ ${ }^{1}$ Klinik für Frührehabilitation und Geriatrie, FEK - Friedrich-Ebert-Krankenhaus Neumünster GmbH, \\ Neumünster, Deutschland \\ ${ }^{2}$ Krankenhaus Hietzing, Wien, Österreich \\ ${ }^{3}$ Krankenhaus Rotes Kreuz, Lübeck, Deutschland
}

\title{
Assessment II
}

\section{Settings, Systematik und Tools}

Bereits ab den 1960er Jahren begann in den USA eine Diskussion um ein um die Selbsthilfefähigkeit erweitertes Gesundheitsverständnis. Man suchte nach Indikatoren, die die heutzutage im „klassischen" Assessment vorhandenen Instrumente nutzten, und führte erste bevölkerungsbezogene, kontrollierte Studien mit großen Stichproben durch: Assessment in der Versorgungsforschung [1].

Assessment soll - laut Rubenstein - in allen Settings gleichermaßen einsetzbar sein. Historisch hatte das Assessment im klinischen Setting begonnen (Warren: Assessment I im letzten Heft). Es wird in der Akutmedizin (Notaufnahme), perioperativ in der Alterstraumatologie bis hin zur Rehabilitation eingesetzt. Assessment kommt in der pflegerischen Überleitung, im Entlassungs- und „care management" vor, in der Physio- und Ergotherapie sowie in der Psychologie/Neuropsychologie. Es wird im Pflegeheim durchgeführt, in der Hausarztpraxis, in Spezialambulanzen (Memory-Clinic) usw. Der Einsatz ist breit, aber: Bestimmte Prinzipien kehren immer wieder [2, 3].

Die Systematik von Assessment umschreibt Rubenstein folgendermaßen: „Unter umfassendem geriatrischen Assessment versteht man einen multidimensionalen und interdisziplinären Prozess mit dem Ziel, die medizinischen, psychosozialen und funktionellen Probleme und Ressourcen des Patienten zu erfassen und einen umfassenden Behandlungsund Betreuungsplan zu entwickeln" (zit. nach [4], S. 165 f.).

\section{Skalen und Instrumente}

Werkzeuge des Assessments sind deren Skalen und Instrumente. Auch erfahrene Ärzte und Geriater erleben immer wieder Überraschungen, z. B. wenn ein Uhrentest völlig unerwartet und überraschend pathologisch ausfällt. Assessmentinstrumente (Tools) müssen standardisiert sein, um Fehlbeurteilungen zu vermeiden. Sie müssen valide für die Fragestellung sein, praktikabel in der Anwendung, zeitsparend und akzeptabel für den zu Untersuchenden. Der Untersucher wird aus den weit mehr als 100 verfügbaren Instrumenten zunächst eine kleinere Anzahl auswählen, mit der er Erfahrungen sammelt und deren Limitationen und Testgütekriterien er kennt (Sensitivität, Spezifität, Decken- und Bodeneffekte etc.).

Eine gute Diskriminationsfähigkeit ist erforderlich, um bei ebenso guter Reproduzierbarkeit („reliability“) Verläufe zuverlässig messen zu können. Der Untersucher benötigt eine gewisse Vorerfahrung, um zunächst das Instrument auswählen zu können, das die vorliegende Domäne und deren Schweregradausprägung für den betreffenden Patienten voraussichtlich am besten abbildet. Nicht nur für Forschungszwecke ist es sinnvoll, Instrumente einzusetzen, die international standardisiert und auch für die übersetzte Sprache validiert sind.

Für den Einsatz von Assessment gab erstmals 1995 die schweizerisch-deutsche „Arbeitsgruppe für Assessment“ (AGAST, [5]) eine Handlungsempfehlung heraus, 2011 die Österreichische
Gesellschaft für Geriatrie und Gerontologie (ÖGGG, [6]). Im Jahr 2012 gründete sich anlässlich des Gerontologie- und Geriatriekongresses in Bonn eine jetzt trinationale und interdisziplinär zusammengesetzte Arbeitsgruppe Assessment aller einschlägigen, deutschsprachigen geriatrisch-gerontologischen Fachgesellschaften.

Der Trend geht dahin, Empfehlungen für die Auswahl und für den Einsatz zunächst einfacher Screenings, z. B. hinsichtlich Kognition, zu geben, denen dann in gestufter Weise vertiefende Untersuchungen mit weiterführenden Instrumenten folgen. Die Domänen Mobilität/ Sturzgefahr, Ernährung/Dysphagie werden derzeit ebenfalls bearbeitet. Weitere Domänen sind in Planung. Kriterien für die Bearbeitung sind u. a.: Setting, Fragestellung, Treffsicherheit der Zuordnung, Aussagekraft, Abklärungsstufe, Schweregrade, Abbildung von Verläufen etc.

Die Arbeitsgruppe wird derzeit von S. Krupp, Lübeck, und U. Sommeregger, Wien, geleitet und tagt jährlich anlässlich der geriatrisch-gerontologischen Kongresse. Die Termine sind den jeweiligen Kongressprogrammen zu entnehmen. Die Arbeitsgruppe ist für alle Mitglieder einer geriatrisch-gerontologischen Fachgesellschaft offen. Die Unterzeichner dieses Editorials laden gerne dazu ein!

Das vorliegende Heft 2 der Zeitschrift für Gerontologie und Geriatrie beginnt aus dem weiten Spektrum unterschiedlichster Settings - gleich mit 2 Beiträgen aus dem wichtigen Setting: „Pflegeheim“. 


\section{Settings}

\section{Pflegeheim}

\section{Deutschland}

Der erste Beitrag von Grebe zu „Resident Assessment Instrument (RAI) Anwendungsoptionen und Relevanz für Deutschland" fokussiert das Pflegeheim. Seit 1990 ist in den USA dieses Assessmentinstrument zur Beurteilung und Dokumentation von Problemen bei Pflegeheimbewohnern gesetzlich vorgeschrieben. Eine Vielzahl weiterer westlicher Länder hat zwischenzeitlich dieses Assessmentsystem ebenfalls übernommen, darunter die Schweiz. Es fokussiert mehrere Dutzend unterschiedlicher Risiko- und Problembereiche und darf als das am besten untersuchte Assessment zur Optimierung der Pflege gelten. Vor allem scheint dieses Assessment einen substanziellen Lernprozess voranbringen zu können. In Deutschland kam das RAI erst in wenigen, kleineren Studien zum Einsatz. Der Beitrag aus Valendar diskutiert die Gefahr der Überregulierung durch Assessment, im Übrigen auch der ökonomischen Überregulierung. Die hier dargelegten Gesichtspunkte sind in der aktuellen Diskussion zur Neudefinition des Pflegebedürftigkeitsbegriffs in Deutschland von höchster Brisanz.

\section{Schweiz}

Der zweite Beitrag von Anliker et al. „RAI

- Resident Assessment Instrument. Geschichte, Resultate und Erfahrungen aus der Anwendung in der Schweiz" vertritt die praktische, d. h. anwenderbezogene Sichtweise, ebenfalls des RAI. Assessment muss „gemacht“ werden, d. h., es bedarf der Motivation und der Bereitschaft der Mitarbeiter zu zuverlässiger sowie standard- und sachgerechter Ausführung. Den erforderlichen Aufforderungscharakter (Impetus) hatte bereits Warren betont. Anliker et al. betonen den Qualitätsgewinn für die Langzeitpflege und schildern den Einsatz von Assessment sehr anschaulich.

\section{Feinmotorik}

Der dritte Beitrag von Krupp et al. „,Timed up and go ' für die Finger in Form des 20-Cents-Tests. Psychometrische Gütekriterien eines einfachen FeinmotorikPerformance-Tests" befasst sich mit den für das Alltagsleben so wichtigen feinmotorischen Fähigkeiten. Bis heute existiert dafür so gut wie kein standardisiertes Assessmentinstrument. Es ist nicht schwierig vorherzusagen, dass - wenn nur endlich ein entsprechendes Instrument verfügbar ist - dieses auch rasch Verbreitung finden wird. Krupp et al. haben ein derartiges Tool konstruiert und unterziehen es hiermit den ersten Validierungsuntersuchungen. Es ist in den verschiedenen Settings einfach einzusetzen, gut zu "handhaben“ und v. a. „lebensnah“. Nicht zuletzt ist es verbunden mit dem Impetus: Vernachlässigt nicht die oberen Extremitäten.

\section{Onkologie}

Der vierte Beitrag von Denkinger et al. „Predicting fatigue in older breast cancer patients receiving radiotherapy - a head to head comparison of established assessments" ist ein sehr interessantes Beispiel für den Einsatz von Assessment in der Onkologie. Es wird u. a. versucht, Zusammenhänge zwischen einem „Comprehensive geriatric assessment“ (CGIA)Instrument und Outcome-Variablen darzustellen und daraus Vorhersagewerte, hier im speziellen Fall für „fatigue“, zu errechnen. Die „Frailty“-Kriterien nach Fried kommen ebenfalls zum Einsatz. Hier besteht allerdings das Problem der noch nicht abgeschlossenen Operationalisierung, noch fehlender "Cut-off“Punkte und damit der Standardisierung, die für ein Assessmentinstrument zwingend zu fordern sind. Der Forschungsbedarf für dieses enorm wichtige Thema ist offenkundig.

Mit einer der schönsten Definition des Assessment, die von H.B. Stähelin, Basel, stammt, wollen wir Ihnen auf dieses Heft ebenfalls Geschmack machen: „Assess- ment heißt, Ursachenknäuel zu entwirren sowie präventiv und therapeutisch $\mathrm{zu}$ intervenieren. "Und: Besuchen Sie auf einem der nächsten geriatrisch-gerontologischen Jahreskongresse doch einmal die Arbeitsgruppe Assessment!

Ihre

W. Hofmann

S. Krupp

U. Sommeregger

\section{Korrespondenzadresse}

PD Dr. W. Hofmann
Klinik für Frührehabilitation
und Geriatrie
FEK - Friedrich-Ebert-
Krankenhaus Neumünster
GmbH, Friesenstr. 11
24534 Neumünster
werner.hofmann@fek.de

\section{Einhaltung ethischer Richtlinien}

Interessenkonflikt. W. Hofmann, U. Sommeregger und S. Krupp geben an, dass kein Interessenkonflikt vorliegt.

\section{Literatur}

1. Branch LG (1998) Das geriatrische Assessment. In: Steinhagen-Thiessen E (Hrsg) Das geriatrische Assessment. Schattauer, Stuttgart, S 3-28

2. Rubenstein LZ (1998): Wem nutzt das geriatrische Assessment? In: Steinhagen-Thiessen E (Hrsg) Das geriatrische Assessment. Schattauer, Stuttgart, S29-82

3. Rubenstein LZ, Stuck A, Siu A et al (1991) Impacts of geriatric evaluation and management prgrams on defined outcomes. Overview of evidence. J Am Geriatr Soc 39:8-16

4. Nikolaus T (2000) Konzept des geriatrischen Assessments. In: Nikolaus T (Hrsg) Klinische Geriatrie. Springer, Berlin Heidelberg New York Tokio, S 161188

5. Bach M, Hofmann W, Nikolaus T (1995) Geriatrisches Basisassessment. Handlungsanleitungen für die Praxis. Arbeitsgruppe geriatrisches Assessment (AGAST). MMV-Verlag, München

6. Sommeregger U (2011) Assessment. Österreichische Gesellschaft für Geriatrie und Gerontologie (ÖGGG). Eigenverlag, Wien 